AlPER, T. \& GiLLEs, N. E. (1960). J. gen. Microbiol. 22, 113-128

\title{
The Relationship between Growth and Survival after Irradiation of Escherichia coli Strain B and two Resistant Mutants
}

\author{
By TIKVAH ALPER AND N. E. GILLIES \\ Medical Research Council, Experimental Radiopathology \\ Research Unit, Hammersmith Hospital, London, W. 12
}

SUMMARY: Faster growth of Escherichia coli strain B cells is observed in media which dontain factors previously found to increase the numbers killed by radiation. Incubation in the presence of metabolic inhibitors after irradiation allows many more irradiated cells to originate colonies. Holding the cells on complete media at low temperatures after irradiation causes more irradiated cells to die, an exception to the principle that greater survival of strain B is associated with slower growth. The difference in the radiosensitivity of strain $B$ and two resistant mutants, strains $B / r$ and Bpr 5, depends on differences in their utilization of organic nutrients, since: (i) all three strains have about the same sensitivity if they are plated on a minimal medium; (ii) if strains $B$ and $B / r$ are treated with chloramphenicol immediately after ultraviolet, the survival of strain $B$ increases, and that of strain $B / r$ decreases, both reaching the same level as if they had been plated immediately on minimal medium; (iii) if irradiated cells of strains $B$ and $B / r$ are incubated on nutrient medium for a short period before chloramphenicol treatment, the survival of strain B roughly equals that of the resistant mutants, while that of $B / r$ is unaffected. When logarithmic phase broth-grown cells of all three strains are exposed to visible light after ultraviolet, only strain B is 'photoreactivated', and then only when viable counts on nutrient medium are compared, the counts on synthetic medium being unaffected by the visible light.

Many strains of micro-organisms have been shown to vary in their response to radiation according to the conditions of culture after irradiation. Escherichia coli strain B seems particularly variable in this respect, and numerous procedures have been reported to bring about 'recovery' or 'reactivation' of irradiated cells, e.g. holding in suspension before plating (Roberts \& Aldous, 1949) or incubation at high temperatures (Stein \& Meutzner, 1950). We have suggested (Alper \& Gillies, 1958) that a feature common to these procedures is their ability to slow down metabolism after irradiation, so preventing the occurrence of death due to 'unbalanced growth' (Heinmets \& Kathan, 1954; Barner \& Cohen, 1956). Thus the greatest effect of a given dose of radiation was observed in conditions which were normally optimal for the growth of these cells (Alper \& Gillies, 1958).

This hypothesis has been further tested by investigating in greater detail the rate of growth of Escherichia coli strain B in various conditions in which the survival after irradiation differed. The effect of treating irradiated cells with metabolic inhibitors has also been studied.

The response of irradiated Escherichia coli strain B cells to factors (such as the concentration of chloride and peptone) in the medium is in marked 
contrast to that of two 'radio-resistant' mutants, viz. strain B/r (Witkin, 1947) and a strain isolated in this laboratory and designated strain Bpr5. As reported previously (Alper \& Gillies, 1958), the survival of $E$. coli strain B/r, in contrast with the behaviour of strain $B$, was not affected by the presence of chloride in the medium, or by increasing the peptone content of a complete medium beyond $5 \mathrm{~g} . / 1$. Stapleton, Sbarra \& Hollaender (1955) reported that cells of strain $\mathbf{B} / \mathbf{r}$ showed lower survival after $\mathbf{X}$-irradiation when they were plated on minimal than on complete medium. Comparative studies of the response of $E$. coli $B$ and the two mutants, strain $B / \mathbf{r}$ and strain $B$ pr 5 , to conditions imposed after irradiation have now been extended.

\section{MATERIALS AND METHODS}

Strains. The strains Escherichia coli strain B and strain B/r were the same as we used previously (Alper \& Gillies, 1958). A new resistant mutant of strain B, designated strain Bpr 5, was selected after irradiating a population of strain B with an ultraviolet (u.v.) dose such that 15 colonies were formed from a total of about $1.5 \times 10^{7}$ cells plated. All the colonies were tested and only one strain was found to be resistant to radiation.

Media and chemicals. The nutrient media used (Oxoid Blood Agar Base, Oxoid Nutrient Broth), the methods of adjusting their $\mathrm{pH}$ values, and the addition of various other components, e.g. Oxoid $\mathrm{NaCl}$ and Oxoid Peptone, have previously been described (Alper \& Gillies, 1958). In some experiments we used a minimal salts medium devised by Davis (quoted by Lederberg, 1950). This consisted of $27 \cdot 5 \mathrm{~g}$. $\mathrm{KH}_{2} \mathrm{PO}_{4}, 57 \cdot 5 \mathrm{~g}$. $\mathrm{K}_{2} \mathrm{HPO}_{4}, 2 \cdot 35 \mathrm{~g} . \mathrm{Na}_{3}$ citrate. $2 \mathrm{H}_{2} \mathrm{O}, 0.625 \mathrm{~g} \cdot \mathrm{MgSO}_{4} \cdot 7 \mathrm{H}_{2} \mathrm{O}$ and $5 \cdot 0 \mathrm{~g}$. $\left(\mathrm{NH}_{4}\right)_{2} \mathrm{SO}_{4}$ dissolved in $1 \mathrm{l}$. of distilled $\mathrm{H}_{2} \mathrm{O}$. The solution was diluted 1:5 before use, and glucose, which had been sterilized separately, was added to give a final concentration of $0.8 \%(w / v)$. This medium will be described as 'synthetic medium'. When the medium was required in solid form $1.5 \%(\mathrm{w} / \mathrm{v})$ unwashed Oxoid agar-agar was included. Stock solutions of iodoacetic acid (Roche Products Ltd.), brought to $\mathrm{pH} 7$ with $\mathrm{NaOH}$, and chloramphenicol (Allen and Hanbury Ltd.) were stored at $4^{\circ}$, and diluted to the appropriate concentrations when required.

Preparation of bacteria for irradiation and for growth studies. The method of preparing the cell suspensions has been previously described (Alper \& Gillies, 1958). In every case cells were thrice washed and resuspended in buffer, before irradiating or seeding into fresh media for growth studies. The concentration of culture medium introduced into the fresh medium with the inocula used could not have been as much as $10^{-10} \mathrm{ml} . / \mathrm{ml}$., and was probably considerably less. Methods of seeding the cells on agar or on cellophan carriers have been described (Alper \& Gillies, 1958; Gillies \& Alper, 1959).

Determination of growth curves. The length of the lag phase and the generation time were measured in two ways:

(1) About $10^{4}$ organisms were inoculated into $10 \mathrm{ml}$. of liquid medium, kept at $37^{\circ}$ in a water-bath. At intervals samples were removed for making viable counts. Oxoid 'Blood Agar Base' plates were used routinely for studying the 
growth of unirradiated cells, since no difference in viable count could be detected on any of the solid growth media used. After irradiation, however, the viable count of Escherichia coli strain B and its mutants was markedly affected by the medium (Roberts \& Aldous, 1949; Stapleton, Sbarra \& Hollaender, 1955; Weatherwax, 1956; Alper \& Gillies, 1958); the growth of survivors of irradiation was therefore studied by using solid and liquid media of the same composition.

(2) In some experiments it was necessary to establish the length of the lag phase and generation time of cells which had been seeded on the surface of cellophan carriers lying on solid medium (Alper \& Gillies, 1958). 0.004 ml. of a bacterial suspension of appropriate concentration was applied by means of an 'Alga' micro-syringe (Burroughs Wellcome and Co., London) to each of several narrow strips of cellophan $(40 \mathrm{~mm} . \times 6 \mathrm{~mm}$.) lying on agar gel containing no nutrients. After irradiation the strips were transferred to the required solid medium previously warmed to $37^{\circ}$, and were incubated at $37^{\circ}$. At suitable intervals two strips were removed and dropped into McCartney bottles containing $1 \mathrm{ml}$. of $\mathbf{0 . 0 6 7} \mathrm{M}$-phosphate buffer, pH 7 . The bottles were shaken for $1 \mathrm{~min}$. and viable counts were made of the suspensions so obtained.

Use of metabolic inhibitors. We have previously described a technique whereby cells can be exposed to the action of metabolic inhibitors for defined periods (Gillies \& Alper, 1959). In all experiments with chloramphenicol the concentration was $5 \mu \mathrm{g} . / \mathrm{ml}$. in Oxoid 'Blood Agar Base'. In experiments with sodium iodoacetate it was found necessary to use that concentration which would just inhibit the formation of visible colonies. This concentration depended on the medium used; the higher the nutritive value of the medium, the more iodoacetate was required. The range of concentrations used varied from $0.001 \mathrm{M}$ to $0.01 \mathrm{M}$.

Experiments were also performed in which the rate of metabolism was reduced for a limited period by incubating cells in nutrient broth under anoxia. A sample of the cell suspension was added to $5 \mathrm{ml}$. of broth contained in a vessel through which gas could be bubbled, and from which samples could be removed without admitting air (Alper, 1955). Immediately after inoculation oxygen-free nitrogen was passed through the broth, previous experiments having shown that in these conditions the oxygen content was reduced by a factor of $10^{4}$ within 2 min. after the nitrogen started passing through the vessel. Simultaneously, equivalent suspensions were prepared in McCartney bottles, and these were aerated by rotating them on a sloping turn-table at 78 r.p.m. All four suspensions were incubated at $37^{\circ}$, and samples were removed at intervals to determine the viable counts.

Methods and sources of irradiation. The source and dose rate of the u.v. were as previously described (Alper \& Gillies, 1958). X-irradiations were carried out under defined conditions of gas equilibration ( $100 \%$ oxygen or no oxygen), the vessels used having been described (Alper, 1955). The source of $\mathrm{X}$-rays was a Solus-Schall $300 \mathrm{kV}$. constant potential industrial $\mathrm{X}$-ray unit operating at $250 \mathrm{kV}$., and $10 \mathrm{~mA}$., with no added filtration. The dose rate in the vessels was $1100 \mathrm{rad} . / \mathrm{min}$. 


\section{RESULTS}

\section{Growth of Escherichia coli strain B in various media}

Previous experiments had shown that several factors in the plating medium affected survival after irradiation (Alper \& Gillies, 1958). We therefore studied the effects of these factors on the growth rate of non-irradiated cells, and in some cases of cells which survived irradiation.

(i) Organic factors. Figure 1 shows the lag phase and generation time of strain $B$ in Oxoid and Difco nutrient broth, containing respectively 25 and $8 \mathrm{~g}$./l. of organic nutrients, and in synthetic medium, while Fig. 2 shows that

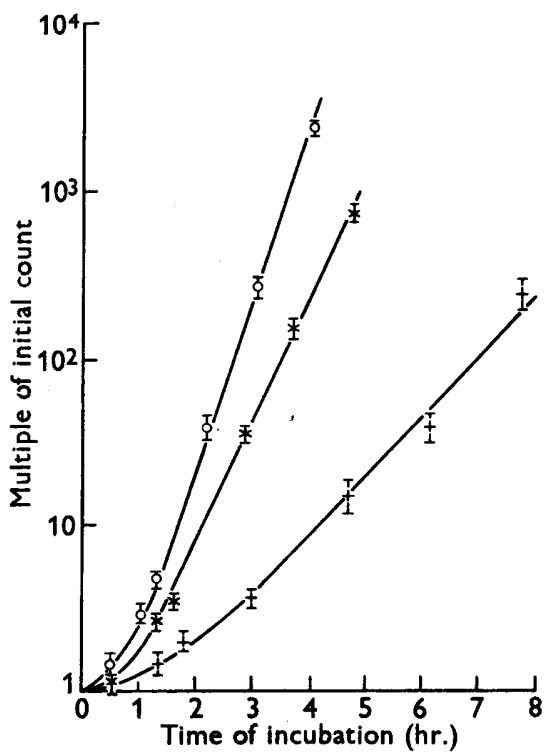

Fig. 1

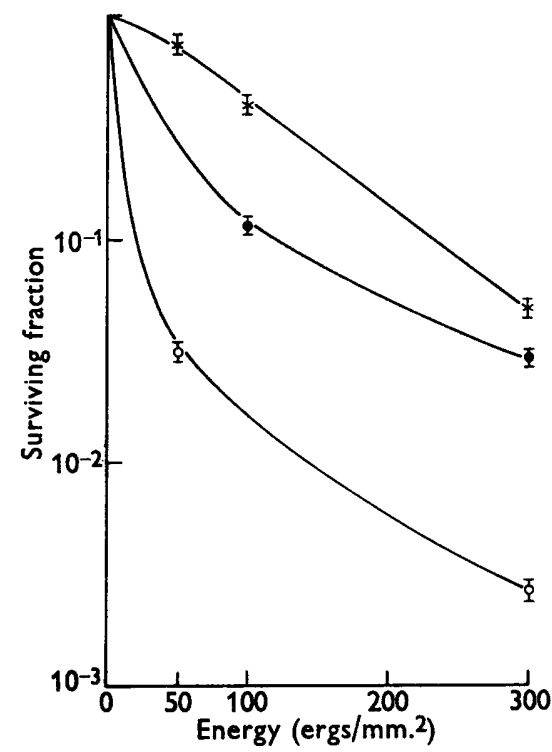

Fig. 2

Fig. 1. Growth of non-irradiated Escherichia coli strain B (harvested in the logarithmic phase from Oxoid broth). O, Oxoid broth; $\times$, Difco broth; + , synthetic medium.

Fig. 2. Survival of Escherichia coli strain B (harvested in the logarithmic phase from liquid synthetic medium), after exposure in buffer suspension to ultraviolet light. $\times$, synthetic medium;, Difco agar; $O$, Oxoid agar.

survival after u.v. was lowest on the medium on which growth was fastest. The experiments illustrated by Fig. 2 were carried out with cells which had been grown in synthetic medium before irradiation, demonstrating that the relatively high survival on synthetic medium did not depend on the adaptation to this medium of broth-grown cells.

Peptone contains a dialysable factor which reduces survival after irradiation (Alper \& Gillies, 1958), and we found that growth rate in synthetic medium or Difco nutrient broth was increased by adding peptone dialysate. This factor has not yet been identified, but various substances when added to synthetic medium had a similar effect. Figures $3 a$ and $3 b$ illustrate the effects 
of adding a mixture of ribonucleotides, prepared by degrading yeast RNA with $0.05 \mathrm{~N}-\mathrm{NaOH}$. The addition of individual purines or pyrimidines, separately or mixed together, did not affect survival.

(ii) Addition of NaCl. After irradiation the survival of Escherichia coli strain $\mathrm{B}$ was reduced if $\mathrm{Cl}^{-}$was added to nutrient media (Alper \& Gillies, 1958), and addition of $\mathrm{NaCl}$ to media containing organic nutrients reduced both the lag period and generation time of non-irradiated and irradiated cells (Fig. 4). However, addition of $\mathrm{NaCl}$ to synthetic medium altered neither growth rate nor survival (Figs. $3 a$ and $3 b$ ).

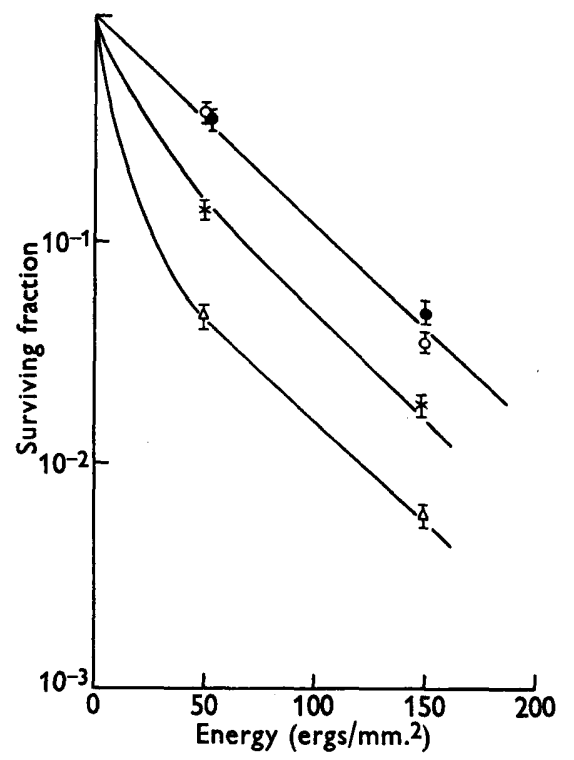

$\boldsymbol{a}$

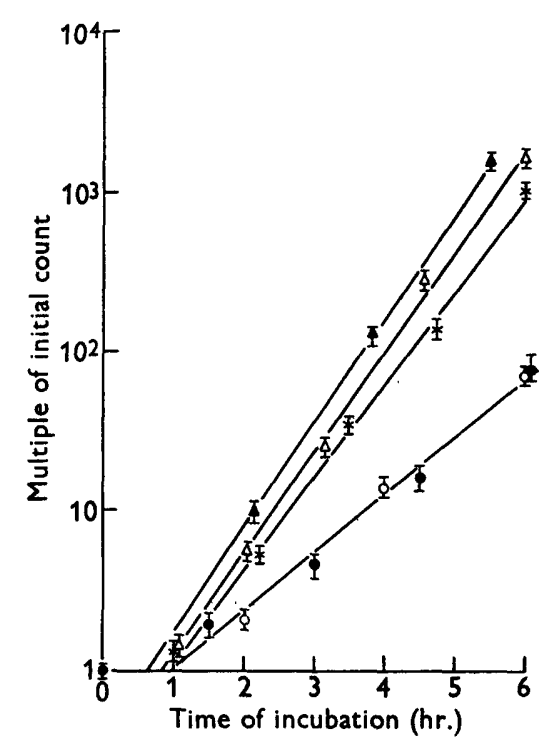

$\boldsymbol{b}$

Fig. 3a. Survival of Escherichia coli strain B (logarithmic phase, Oxoid broth) after exposure, on cellophan carriers, to ultraviolet light. $O$, synthetic medium; 9 , synthetic medium $+\mathrm{NaCl}, 5 \mathrm{~g} . / 1 . ; \times$, synthetic medium + degraded yeast $\mathrm{RNA}, 1.56$ g./1., + NaCl, 5 g./l.; $\triangle$, synthetic medium + degraded yeast $\mathrm{RNA}, 6 \cdot 24 \mathrm{~g} . / \mathrm{l}$., + $\mathrm{NaCl}, 5 \mathrm{~g} . / \mathrm{l}$.

Fig. 3b. Growth of non-irradiated Escherichia coli strain B (stationary phase, Oxoid broth). $O$, synthetic medium; $O$, synthetic medium $+\mathrm{NaCl}, 5 \mathrm{~g} . / 1$; $\times$, synthetic medium + degraded yeast RNA, 0.79 g./l.; $\triangle$, synthetic medium + degraded yeast RNA, 2.37 g./l., $+\mathrm{NaCl}, 5$ g./l.; $\Delta$, synthetic medium + degraded yeast RNA, 9.48 g./1.

When the concentration of organic nutrients was high enough to give a generation time of $18 \mathrm{~min}$., the addition of $\mathrm{Cl}^{-}$did not reduce this further, but it did reduce survival after irradiation. The dependence of the survival of irradiated Escherichia coli strain $\mathrm{B}$ on the concentration of $\mathrm{NaCl}$ added to Difco nutrient agar is illustrated by Fig. 5 .

(iii) $\mathrm{pH}$. It was observed that survival after irradiation was less, the greater the $\mathrm{pH}$ value of the plating medium, in the range 6.0-9.0 (Weatherwax, 1956; Alper \& Gillies, 1958). Non-irradiated cells of Escherichia coli strain B grew in Oxoid nutrient broth at $\mathrm{pH} 6.3$ and 7.5 with generation times of 
22 and 20 min. respectively, though the lag phases did not differ (Fig. 6). Despite the small differences in generation times, there was considerable difference in the survival after irradiation on these media. In order to reduce the surviving fraction to $10 \%, 1.53$ times the dose of $u . v$. was required when the more acid medium was used.

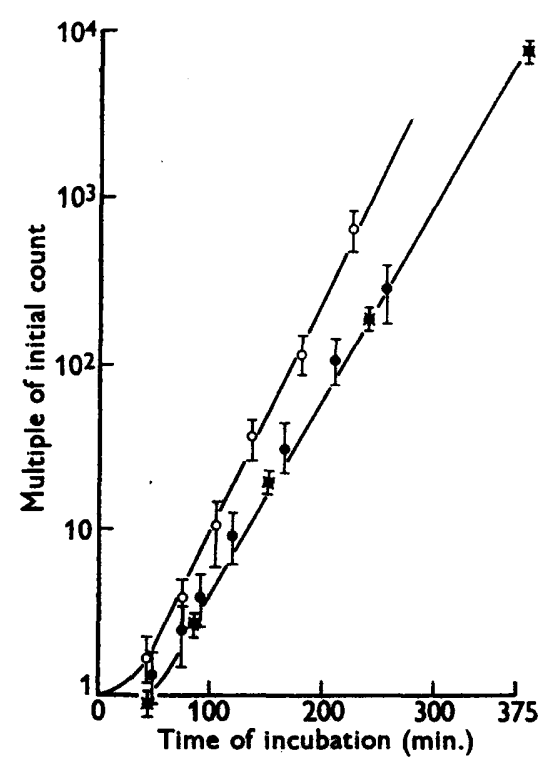

Fig. 4

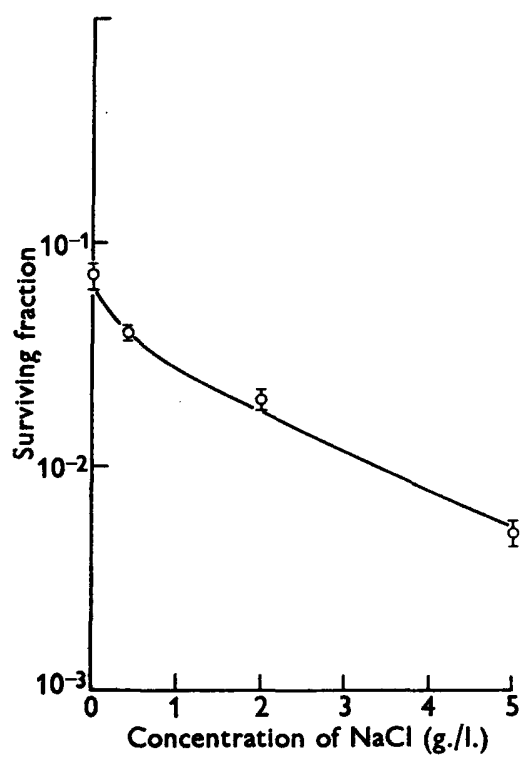

Fig. 5

Fig. 4. Growth of non-irradiated Escherichia coli strain B (logarithmic phase, Oxoid broth) in Difco nutrient broth $(\mathrm{pH} \mathrm{7 \cdot 5)}$.,$\cdots$, containing no $\mathrm{NaCl}$; $O$, containing $\mathrm{NaCl}$ (5 g./1.).

Fig. 5. Effect of concentration of $\mathrm{NaCl}$ added to Difco nutrient agar (pH 6.6) on survival of Escherichia coli strain B (logarithmic phase, Oxoid broth) after exposure, in buffer suspension, to $54 \mathrm{ergs} / \mathrm{mm}^{2} \mathrm{u} . \mathrm{v}$. light.

\section{Effect on Escherichia coli strain $B$ of withholding nutrients after irradiation}

The number of irradiated cells which give rise to colonies increases with increasing the time lapse between irradiation and contact with growth medium, until maximum 'restoration' occurs (Roberts \& Aldous, 1949; Charles \& Zimmerman, 1956). After a dose of $27 \mathrm{ergs} / \mathrm{mm} .^{2}$, cells stored in buffer for $45 \mathrm{~min}$. before plating gave rise to 10 times as many colonies as those plated immediately, and their lag period after inoculation into Oxoid broth was 75 min longer than that of cells inoculated immediately, whereas nonirradiated cells similarly treated had equal lag periods. As shown by Fig. 7, the generation time of the irradiated cells was the same for both groups, and was shorter than that of the controls, as has previously been observed after X-irradiation (Alper, 1957).

The increased period of lag observed when irradiated cells were stored in buffer may be due to the leaking out of certain compounds (cf. Webb \& 
Loofbourow, 1947; Loofbourow, 1947; Loofbourow, Oppenheim-Errara, Loofbourow \& Yeats, 1947; Billen, 1957). We observed an increase in the absorption of the suspending buffer at $260 \mathrm{~m} \mu$ the longer the cells were stored after irradiation.

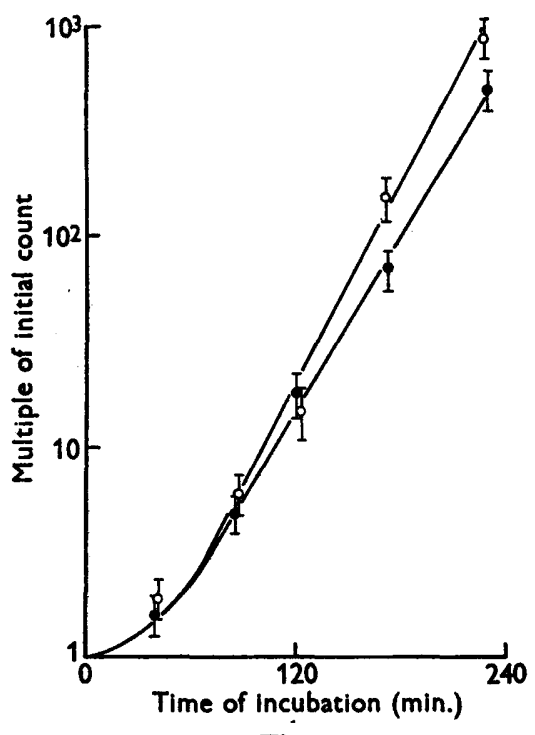

Fig. 6

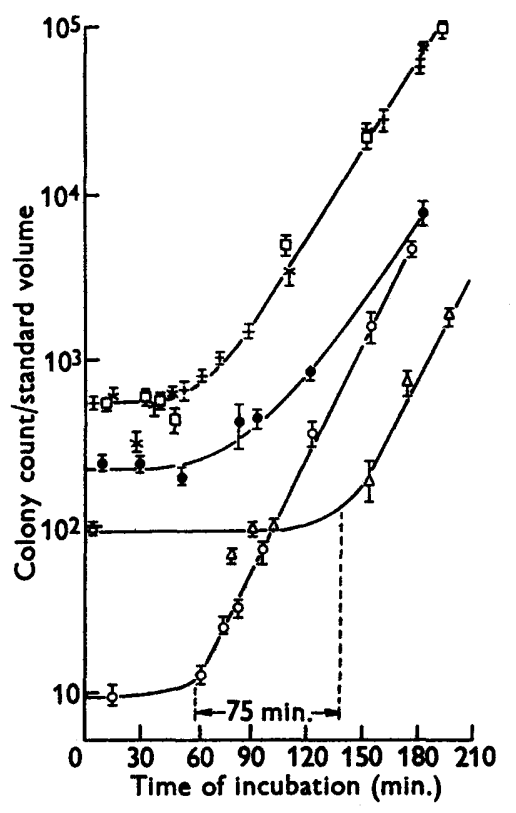

Fig. 7

Fig. 6. Growth of non-irradiated Escherichia coli strain B (logarithmic phase, Oxoid broth).

, Oxoid nutrient broth, pH 6.8; O, Oxoid nutrient broth, pH 7.5.

Fig. 7. Growth of non-irradiated and u.v.-irradiated (27 ergs/mm. ${ }^{2}$ ) Escherichia coli strain B (logarithmic phase, Oxoid broth) in Difco nutrient broth $+\mathrm{NaCl}, 5 \mathrm{~g}$./l. Colony counts made on Difco agar + NaCl, 5 g./. $x$, non-irradiated, inoculated into broth immediately; $\square$, non-irradiated, kept in dark for $45 \mathrm{~min}$. before inoculation into broth; + , non-irradiated, exposed to visible light for $45 \mathrm{~min}$. before inoculation into broth; $O$, irradiated, inoculated into broth immediately; $\Delta$, irradiated, kept in dark for $45 \mathrm{~min}$. before inoculation into broth; $O$, irradiated, exposed to visible light for $45 \mathrm{~min}$. before inoculation into broth. Incubation time from moment of inoculation into nutrient broth.

\section{Effects of metabolic inhibitors on Escherichia coli strain $B$}

Since increased survival after irradiation appeared to be correlated with slow growth, or with an increase in the lag period, it was predicted that metabolic inhibitors would also increase the survival of strain $B$.

\section{(i) Anaerobiosis}

Previous experiments had shown that considerable changes in the numbers of irradiated cells originating colonies could occur within a short period after irradiation (Alper \& Gillies, 1958; Gillies \& Alper, 1959). The respiration rate of Escherichia coli is not reduced to $50 \%$ of normal until the concentration 
of oxygen in the surrounding medium is as low as $2 \cdot 2 \times 10^{-8} \mathrm{M}$ (Longmuir, 1954 ), i.e. less than $10^{-4}$ of the oxygen concentration normally present in water equilibrated with atmospheric air. It was apparent therefore that any effect of anaerobiosis on irradiated cells would be observed to its fullest extent only if rigorously anoxic conditions were imposed very soon after the end of irradiation. This was not possible with conventional methods of producing anoxia in solid media, and we therefore carried out our experiments in broth, although this had the disadvantage that the full extent or the restoration caused by anaerobic conditions was masked when cell division started. Table 1 shows the results of an experiment in which viable counts were made on cells which had been exposed to u.v., and also on non-irradiated cells, incubated in both oxygen-free and in aerated Oxoid broth. With non-irradiated cells, the ratio of cell counts in the oxygen-free and aerated broth remained about one until the end of the lag phase, and then decreased. With irradiated cells, however, the ratio rose to 5 during the period when no division was taking place in the aerated medium. Thus the imposition of anaerobiosis immediately after irradiation brought about a considerable increase in the number of cells which could give rise to colonies.

Table 1. The effect of anaerobiosis on the survival of Escherichia coli strain $B$ (logarithmic phase) inoculated into Oxoid nutrient broth after exposure to $66 \mathrm{ergs} / \mathrm{mm}^{2}$ of ultraviolet light

$\begin{array}{ccc}\text { Time after incubation } & \text { Ratio: } \frac{\text { Viable count in anoxic suspension }}{\text { Viable count in aerobic suspension }} \\ \text { (min.) } & \overbrace{\text { Non-irradiated }}^{\text {Irradiated }} \\ 0 & 1 \cdot 19 & 0 \cdot 70 \\ 60 & 1 \cdot 22 & 4 \cdot 66 \\ 120 & 0 \cdot 69 & 4 \cdot 64\end{array}$

The times of the first division of the non-irradiated and irradiated cells in the aerated medium were about 35 and $85 \mathrm{~min}$. respectively.

\section{(ii) Iodoacetate}

Incubation with iodoacetate, which inhibits carbohydrate metabolism, had been found by Wainwright \& Mullaney (1953) and Wainwright \& Nevill (1955) to increase the survival of certain micro-organisms after u.v.-irradiation. We obtained some restoration of strain $B$ by incubating for a period on medium containing iodoacetate, then transferring the cells to fresh medium (Fig. 8). No effect of iodoacetate was observed unless the cells had been incubated for a period before irradiation (at least $2 \mathrm{hr}$.) in a medium containing no carbon source.

The concentration of iodoacetate was very critical. If it was too low, control cells left overnight on the iodoacetate medium grew into colonies. If there was just enough iodoacetate to inhibit colony formation, the control cells started dying after about an hour, and the period of contact of irradiated cells with the iodoacetate was therefore limited to this interval, which was 
probably less than the period required to effect maximum restoration of the irradiated cells.

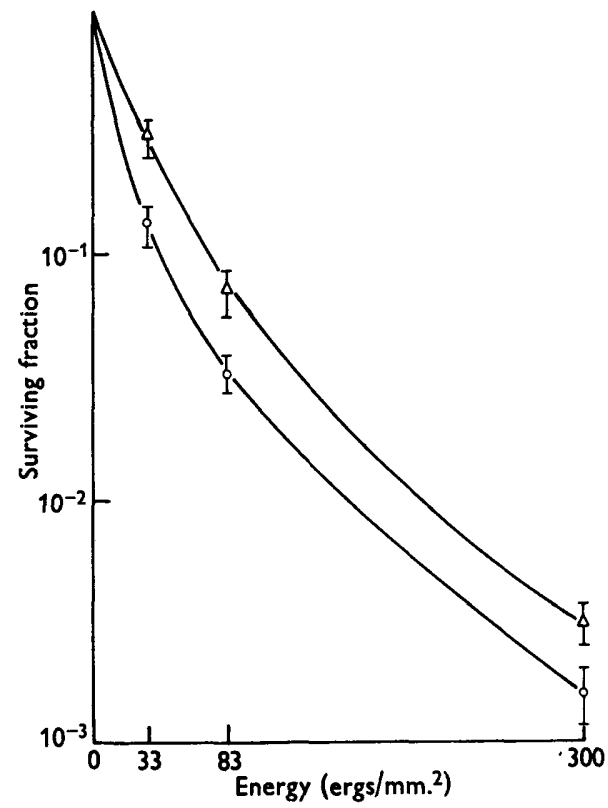

Fig. 8. Survival of Escherichia coli strain B (stationary phase, Oxoid broth) after exposure, on cellophan carriers, to ultraviolet light. $O$, on Oxoid agar; $\triangle$, on Oxoid agar $+\mathrm{Na}$ iodoacetate $(0.01 \mathrm{M})$ for $1.3 \mathrm{hr}$. before transfer to Oxoid agar.

\section{(iii) Chloramphenicol}

This was the most effective of the metabolic inhibitors we used. After Xor u.v.-irradiation, the numbers of survivors increased, with increasing time of contact with chloramphenicol, until a maximum was reached which was about the same as if the cells had been plated immediately on synthetic medium. Highest survival was obtained if the irradiated cells were incubated for a period, less than the time to first division, at $37^{\circ}$ on Oxoid 'Blood Agar Base', then transferred to plates of the same medium containing $5 \mu \mathrm{g}$. chloramphenicol/ml., and finally incubated on Oxoid 'Blood Agar Base' (Gillies \& Alper, 1959). Figures 9 and 10 show how survival of strain $B$ varied with dose of $\mathrm{X}$ - and u.v.-irradiation, with and without the treatment which gave maximum survival.

The survival of strain B was increased by chloramphenicol treatment only if it was used with complete medium. If synthetic medium containing chloramphenicol was used for plating broth-grown cells after irradiation, their viability decreased with time of incubation on the chloramphenicol medium. If the same medium was used to treat cells grown in synthetic medium before irradiation, no change in their survival was observed. 


\section{Comparisons of strain $B$ with resistant mutants after irradiation}

\section{Growth rate in liquid media}

All three strains grew at the same rate in complete media. When logarithmicstage cells were inoculated into synthetic medium, the lag phase and generation time of strain $B / r$ cells were slightly less than those of the other two strains.

\section{Effect of organic nutrients after irradiation}

Strain $B$ is much more sensitive than strain $B / r$ to u.v. or ionizing radiation if nutrient medium is used for plating (Witkin, 1947). It has been shown above that more strain $B$ cells survive each dose, if synthetic medium is used, whereas fewer strain $B / r$ cells survive $X$-irradiation if they are plated on synthetic medium (Stapleton, Sbarra \& Hollaender, 1955). We found that the

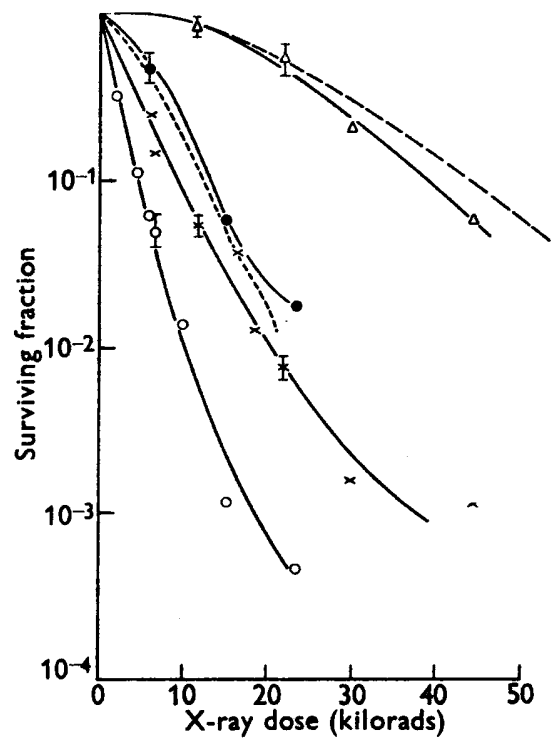

Fig. 9

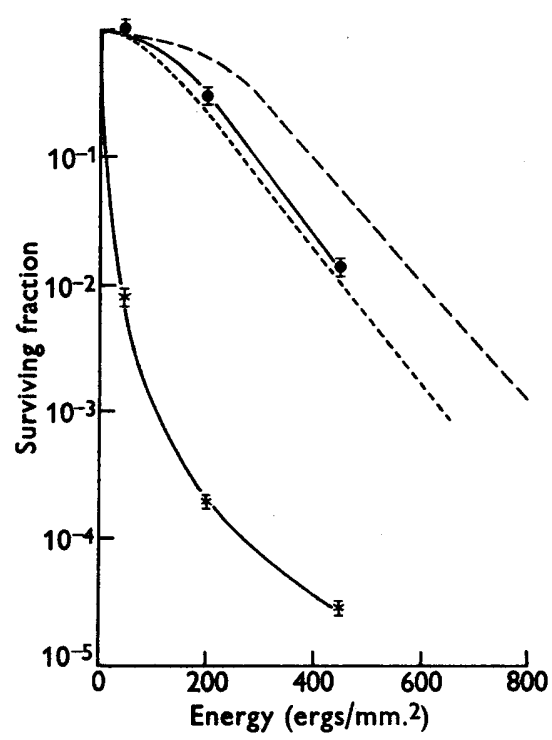

Fig. 10

Fig. 9. Survival of Escherichia coli strain B (stationary phase, Oxoid broth) after exposure in buffer suspension to $X$-rays under oxygenation and under anoxia. The subsequent growth was on cellophan carriers. $O$, irradiated under oxygenation, grown on Oxoid agar; $x$, irradiated under anoxia, grown on Oxoid agar; 0 , irradiated under oxygenation, placed on Oxoid agar + chloramphenicol, $5 \mu \mathrm{g} . / \mathrm{ml}$., under conditions which gave maximum 'restoration', and then grown on Oxoid agar; $\triangle$, irradiated under anoxia, placed on Oxoid agar + chloramphenicol, $5 \mu \mathrm{g} . / \mathrm{ml}$., and then grown on Oxoid agar. The curve composed of long dashes and that composed of short dashes indicate respectively the survival on Oxoid agar of $E$. coli $B / \mathbf{r}$ after $X$-irradiation under anoxia, and of E. coli Bpr 5 after X-irradiation under oxygen.

Fig. 10. Survival of Escherichia coli strain B (logarithmic phase, Oxoid broth) after exposure on cellophan carriers to u.v. light. $x$, on Oxoid agar; $O$, on Oxoid agar +chloramphenicol, $5 \mu \mathrm{g} . / \mathrm{ml}$., under conditions which gave maximum 'restoration', and then transferred to Oxoid agar. The curve composed of long dashes indicates the survival of $E$. coli $B / \mathrm{r}$ and that composed of short dashes the survival of $E$. coli Bpr 5, on Oxoid agar after irradiation with u.v. light. 
greatest effect of medium on all three strains occurred when logarithmicphase cells were exposed to u.v., and in these conditions, the sensitivity of all the strains was the same if synthetic medium was used. As shown by Figs. 11 and 12, the survival curves for the resistant strains plated on nutrient medium were sigmoid, and such curves are often assumed to indicate that there is a 'multiple target' for the irradiation damage (Witkin, 1947; Zelle \& Hollaender, 1955). However, if the cells were plated on synthetic medium the survival curves were exponential, with a slope equal to that of the curves taken on nutrient medium. Thus the difference in sensitivity between strains $B$ and the resistant mutants, as well as the 'multiple-target' nature of the survival curves characteristic of the latter, arose from the effect on the cells of the nutrients supplied after irradiation.

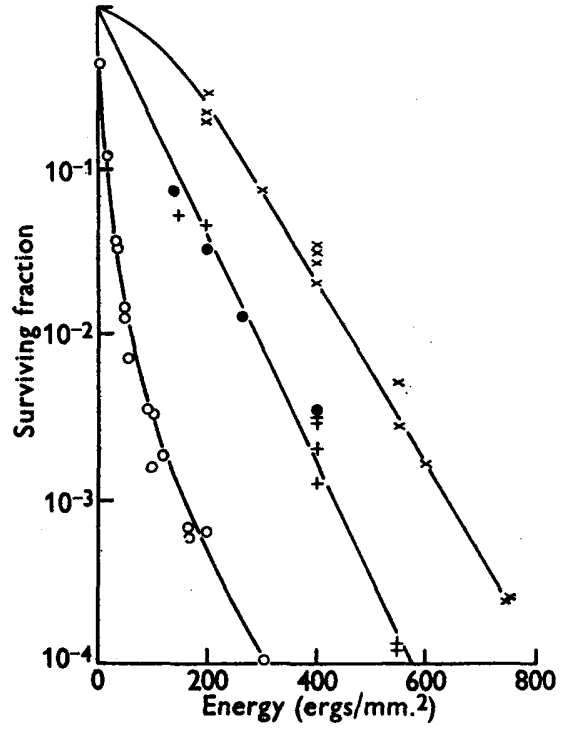

Fig. 11

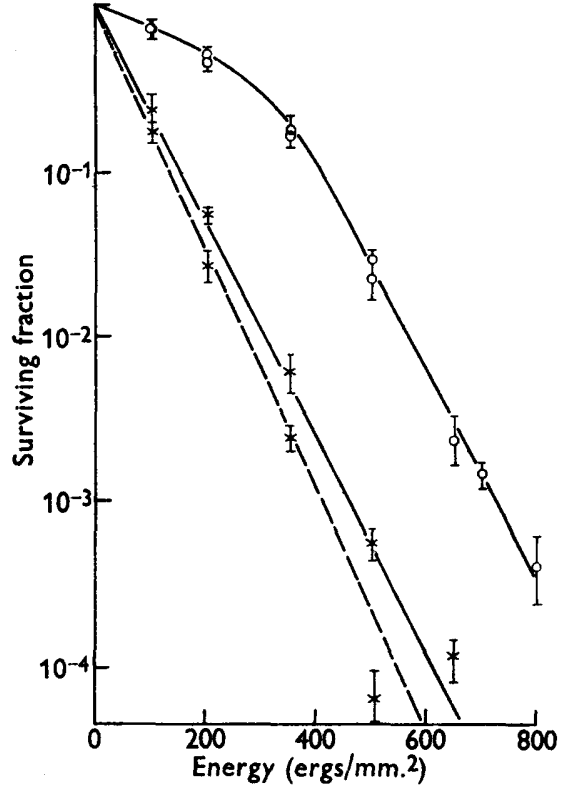

Fig. 12

Fig. 11. Survival of Escherichia coli strain B (logarithmic phase, Oxoid broth) and E. coli Bpr 5 (logarithmic phase, Oxoid broth) after exposure, on cellophan carriers, to u.v. light. $O, E$. coli B on Oxoid agar;,$E$. coli B on synthetic medium; $\times, E$. coli Bpr 5 on Oxoid agar;,$+ E$. coli Bpr 5 on synthetic medium.

Fig. 12. Survival of Escherichia coli strain B/r (logarithmic phase, Oxoid broth) after exposure, on cellophan carriers, to u.v. light: pooled results, two experiments. $O$, Oxoid agar; $\times$, synthetic medium. The dashed curve indicates the survival of $E$. coli $B$ on synthetic medium after exposure to u.v. light under the same conditions.

\section{Effect of incubation temperature after irradiation}

Several reports have been made of the effects of incubation temperature on the survival of strain B after irradiation (Anderson, 1951; Buzzell, 1956; Stein \& Meutzner, 1950). Stapleton, Billen \& Hollaender (1955) reported that strain $B / r$ survived $X$-rays to the greatest extent if the cells were held at 
$18^{\circ}$ for several hours after $\mathrm{X}$-irradiation. We have confirmed this result, but have found that after u.v. survival is slightly reduced by the same treatment. With strain B, the effects of temperature did not vary much with the type of radiation used. Survival after $\mathbf{X}$-rays or u.v. was least if the cells were incubated at $20^{\circ}$, which was roughly the temperature which gave maximum survival of B/r after $X$-rays. The results of these studies are shown in Table 2.

The reduction in the survival of irradiated cells of strain $B$ held at temperatures below $37^{\circ}$ is an exception to the principle that increased survival of this strain occurs in conditions which slow growth.

Table 2. Comparison between doses of $X$-or ultraviolet-irradiation to give $10 \%$ survival if cells are held at various temperatures after irradiation

\begin{tabular}{|c|c|c|c|c|}
\hline \multirow[b]{3}{*}{ Strain } & \multirow{3}{*}{$\begin{array}{c}\text { Holding } \\
\text { temp. } \\
\left({ }^{\circ} \mathrm{C}\right)\end{array}$} & \multicolumn{3}{|c|}{ Ratios of doses required } \\
\hline & & \multicolumn{2}{|c|}{ X-rays } & \multirow[b]{2}{*}{ Ultraviolet } \\
\hline & & Anoxic & Oxygenated & \\
\hline E. coli B (logarithmic phase) & $\begin{array}{r}4 \\
\mathbf{2 0} \\
\mathbf{3 0} \\
\mathbf{3 7}\end{array}$ & $\begin{array}{l}- \\
\mathbf{0 . 3 8} \\
0.52 \\
1 \cdot 0\end{array}$ & $\begin{array}{l}0.85 \\
0.34 \\
0.46 \\
1.0\end{array}$ & $\begin{array}{l}1.51 \\
0.52 \\
0.72 \\
1.0\end{array}$ \\
\hline E. coli $\mathbf{B} / \mathbf{r}$ (stationary phase) & $\begin{array}{l}18 \\
37\end{array}$ & $\begin{array}{l}1 \cdot 86 \\
1 \cdot 0\end{array}$ & $\begin{array}{l}2 \cdot 32 \\
1 \cdot 0\end{array}$ & $\begin{array}{l}0.81 \\
1.0\end{array}$ \\
\hline E. coli Bpr 5 (logarithmic phase) & $\begin{array}{l}\mathbf{2 0} \\
\mathbf{3 7}\end{array}$ & $\begin{array}{l}1 \cdot 0 \\
1.0\end{array}$ & $\overline{1 \cdot 0}$ & $\overline{1.0}$ \\
\hline
\end{tabular}

The doses required when cells are incubated at $37^{\circ}$ have been used as the basis for calculating the ratios.

\section{Effects of visible light after ultraviolet}

Escherichia coli strain B cells exposed to visible light after u.v.-irradiation give rise to more colonies than if they are plated immediately and kept in the dark, a phenomenon which is well known as 'photoreactivation' or 'photorestoration' (Kelner, 1949; Jagger, 1958). However, if synthetic medium was used for plating, the extent of the survival was the same, whether or not the cells were illuminated after u.v.-irradiation, and the survival was greater in these conditions than if the cells were illuminated and then plated on Oxoid 'Blood Agar Base' (Fig. 13). When strains B/r and Bpr 5 cells in the same physiological state were used (i.e. in the logarithmic stage, having been cultured in Oxoid nutrient broth), and exposed to visible light for the same length of time, no increase in survival on complete medium could be detected.

\section{Effects of chloramphenicol after ultraviolet}

When logarithmic-phase cells of strain $B / \mathbf{r}$ were plated immediately on nutrient medium containing $5 \mu \mathrm{g}$. chloramphenicol $/ \mathrm{ml}$., the viable counts decreased with time, until the survival was about the same as if the cells had been plated immediately on synthetic medium. Thus strains $B$ and $B / \mathbf{r}$ appeared to have the same sensitivity if they were treated in this way after irradiation. 
If cells of strain $\mathbf{B} / \mathbf{r}$ were incubated for a short time on nutrient agar before the chloramphenicol treatment, their survival was unchanged. As shown by Figs. 9 and 10, the survival of strain $B$ cells which were treated in this way after u.v. or X-rays was about equal to that of the resistant mutant strains.

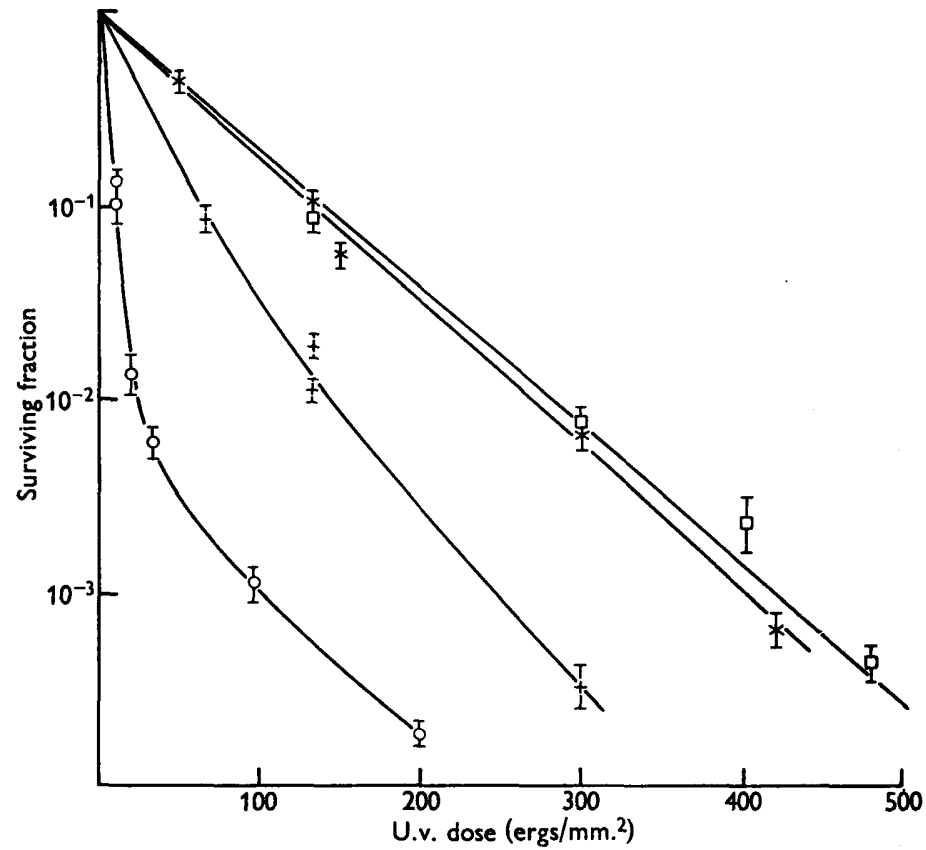

Fig. 13. Survival of Escherichia coli strain B (logarithmic phase, Oxoid broth) after exposure, on cellophan carriers, to u.v. light. $O$, On Oxoid agar; + on Oxoid agar, after exposure to visible light for $1 \mathrm{hr}$.; $\times$, on synthetic medium; $\square$, on synthetic medium after exposure to visible light for $1 \mathrm{hr}$.

\section{DISCUSSION}

The evidence given above demonstrates the close correlation between conditions inhibiting the growth of Escherichia coli strain B after irradiation, and rescue of the cells from radiation damage. This seems to support the hypothesis that the increased survival may be due to the inhibition of certain metabolic processes, which, if allowed to operate normally, would lead to a lethal imbalance in the growth of irradiated cells. The fact that the maximum lethal effect occurs if the cells are held on nutrient medium at about $18^{\circ}$ appears to be in conflict with the hypothesis, but it should be borne in mind that holding at this temperature in the absence of nutrients enables more irradiated cells to survive than if they are immediately incubated with nutrients at $37^{\circ}$. It may be that if nutrients are available to irradiated cells kept at $18^{\circ}$ the differing rate constants of two or more synthetic pathways, one of which is damaged, are such that maximum radiation damage comes to light. There is an analogy between this result and the one previously reported, referring to treatment by chloramphenicol (Gillies \& Alper, 1959). It was found that 
$E$. coli strain B cells irradiated with u.v. while in the logarithmic stage, and incubated immediately afterwards for short periods on nutrient medium containing chloramphenicol, showed more lethal effect of the radiation than untreated cells. Rescue occurred only when the chloramphenicol treatment was prolonged. The initial action of the chloramphenicol on cells in this physiological state was apparently on the synthetic pathway already affected by radiation, and only after longer incubation at $37^{\circ}$ was the second pathway inhibited.

The difference in radiosensitivity between Escherichia coli strain B and its resistant mutants evidently arises from differences in the metabolic pathways leading to cell division, if organic nutrients are present. This is demonstrated by the following facts: $(a)$ The radiosensitivity of the parent strain was about the same as that of the mutants, if judged by the survival of logarithmic-phase cells on synthetic medium. (b) If cells were treated with chloramphenicol immediately after u.v.-irradiation, and then incubated on complete medium, the survival of strain $B$ increased, while that of $B / r$ decreased, both strains reaching a survival level which was about the same as if they had been plated on synthetic medium. (c) If irradiated cells of strain B were incubated on complete medium for a short period before chloramphenicol treatment, and finally incubated on complete medium, survival was about the same as that of the resistant mutants, which were not affected by chloramphenicol treatment given in this way.

The action of visible light after u.v. appears comparable with that of chloramphenicol in some respects. Illumination of irradiated Escherichia coli strain $B$ reduced the lethal effect of u.v. when the cells were plated on complete medium, but not when they were plated on synthetic medium. Exposure to visible light after u.v. irradiation had little or no restoring effect on strains $\mathrm{B} / \mathbf{r}$ or Bpr 5 u.v. in the logarithmic phase.

It has been suggested that the sigmoid survival curves obtained with Escherichia coli strain B/r, and its increased radio-resistance, compared with the parent strain, may be due to some form of polyploidy (see Zelle \& Hollaender, 1955). As shown by Fig. 4, however, the 'multiple target' form of survival curve is obtained after u.v. only if complete medium is used for the viable counts; if synthetic medium is used, the survival curves for logarithmicstage cells are exponential, and the sensitivity is equal to that of the parent strain. The data suggest that a single quantum of energy absorbed in a cell may be sufficient to affect a synthetic pathway, and so kill the cell if it is grown on synthetic medium after irradiation; but when complex growth factors are present in the medium, these offer alternative pathways of synthesis, so that it is necessary for several steps in a synthetic chain to be affected before death of the cell ensues. Such a situation would lead to a sigmoid survival curve, with a final slope equal to that for survival on synthetic medium.

We acknowledge with gratitude the skilful assistance given by Miss Brenda Hodgkins with the experiments. 


\section{REFERENCES}

ALPER, T. (1955). Bacteriophage as indicator in radiation chemistry. Radiat. Res. 2, 119.

Axper, T. (1957). Observations on bacterial growth and morphology shortly after irradiation and some remarks on the oxygen effect. Proc. 5th int. Conf. Radiobiology, p. 90.

ALPER, T. \& GuLIEs, N. E. (1958). 'Restoration' of Escherichia coli strain B after irradiation: its dependence on suboptimal growth conditions. J. gen. Microbiol. $18,461$.

ANDERson, E. H. (1951). Heat reactivation of ultraviolet inactivated bacteria. J. Bact. 61, 389.

Barner, H. D. \& Cohen, S. S. (1956). The relation of growth to the lethal damage induced by ultraviolet irradiation in Escherichia coli. J. Bact. 71, 149.

Biluev, D. (1957). Modification of the release of cellular constituents by irradiated Escherichia coli. Arch. Biochem. Biophys. 67, 333.

BuzzelL, A. (1956). The interrelationships between ultraviolet irradiation and the thermal reactivation of Escherichia coli B. Arch. Biochem. Biophys. 62, 97.

Chardes, R. L. \& Zimmerman, L. N. (1956). Dark reactivation in ultraviolet irradiated Escherichia coli. J. Bact. 71, 611.

Gulres, N. E. \& Alper, T. (1959). Reduction in the lethal effects of radiations on Escherichia coli B by treatment with chloramphenicol. Nature, Lond. 183, 237.

Heinmets, F. \& Kathan, R. H. (1954). Preliminary studies on the mechanism of biological action of ultraviolet irradiation and metabolic recovery phenomenon. Arch. Biochem. Biophys. 53, 205.

JAGGER, J. (1958). Photoreactivation. Bact. Rev. 22, 99.

Kraner, A. (1949). Photoreactivation of ultraviolet-irradiated Escherichia coli with special reference to the dose-reduction principle and to ultraviolet-induced mutation. J. Bact. 58, 511.

Kimball, R. F. \& Garther, N. (1951). The influence of light upon the action of ultraviolet on Paramecium aurelia. J. cell. comp. Physiol. 37, 211.

LATARJET, R. (1954). Spontaneous and induced cell restorations after treatments with ionizing and non-ionizing radiations. Acta radiol., Stockh. 41, 84 .

LEDERBERG, J. (1950). Isolation and characterisation of biochemical mutants of bacteria. Meth. med. Res. 3, 5.

LoNGMUIR, I. S. (1954). Respiration rate of bacteria as a function of oxygen concentration. Biochem. J. 57, 81.

Loofbourow, J. R. (1947). Intracellular hormones. 7. Release of amino acids by damaged living yeast cells. Biochem. J. 41, 119.

Loofbourow, J. R., Oppenhem-Errara, S., Loofbourow, D. G. \& Yeats, C. A. (1947). Intracellular hormones. 8. Release of nucleotides and nucleosides by damaged living cells. Biochem. J. 41, 122.

Roberts, R. B. \& Aldous, E. (1949). Recovery from ultraviolet irradiation in Escherichia coli. J. Bact. 57, 863.

Stapterton, G. E., Bircen, D. \& Hollaender, A. (1955). Recovery of X-irradiated bacteria at sub-optimal incubation temperatures. J. cell. comp. Physiol. 41, 345.

Stapleton, G. E., Sbarra, A. J. \& Hollaender, A. (1955). Some nutritional aspects of bacterial recovery from ionizing radiations. J. Bact. 70,7 .

Stein, W. \& MeUtzaner, I. (1950). Reaktivierung von UV-inaktiviertem Bakterium coli durch Wärme. Naturwissenschaften, 37, 167.

Wainwright, S. D. \& Mullaney, J. (1953). Some effects of metabolic inhibitors upon survival of ultraviolet irradiated Escherichia coli. Experientia, 9, 876. 
Wainwright, S. D. \& Nevilu, A. (1955). Modification of the biological effects of ultraviolet irradiation by post-irradiation treatment with iodoacetate and peptone. J. gen. Microbiol. 12, 1.

WeatherwaX, R. S. (1956). Reactivation of ultraviolet-irradiated Escherichia coli. J. Bact. 72, 329.

WebB, A. M. \& Loofbourow, J. R. (1947). Intracellular hormones. 6. Release and synthesis of factors of the vitamin B complex by damaged living cells. Biochem. J. 41, 114.

WrTKIN, E. M. (1947). Genetics of resistance to radiation in Escherichia coli. Genetics, 32, 221.

Zelue, M. R. \& Hollaender, A. (1955). Effects of radiation on bacteria. In Radiation Biology, ed. A. Hollaender. vol. 2, p. 365. New York: McGraw-Hill Book Co., Inc.

(Received 6 July 1959) 